This item was submitted to Loughborough's Research Repository by the author.

Items in Figshare are protected by copyright, with all rights reserved, unless otherwise indicated.

\title{
Optimization of the felge on parallel bars
}

PLEASE CITE THE PUBLISHED VERSION

http://dx.doi.org/10.1080/14763140802632390

PUBLISHER

(C) Routledge (Taylor \& Francis)

VERSION

AM (Accepted Manuscript)

LICENCE

CC BY-NC-ND 4.0

REPOSITORY RECORD

Hiley, Michael J., Roger Wangler, and Gheorghe Predescu. 2019. "Optimization of the Felge on Parallel Bars". figshare. https://hdl.handle.net/2134/11484. 
This item was submitted to Loughborough's Institutional Repository (https://dspace.lboro.ac.uk/) by the author and is made available under the following Creative Commons Licence conditions.

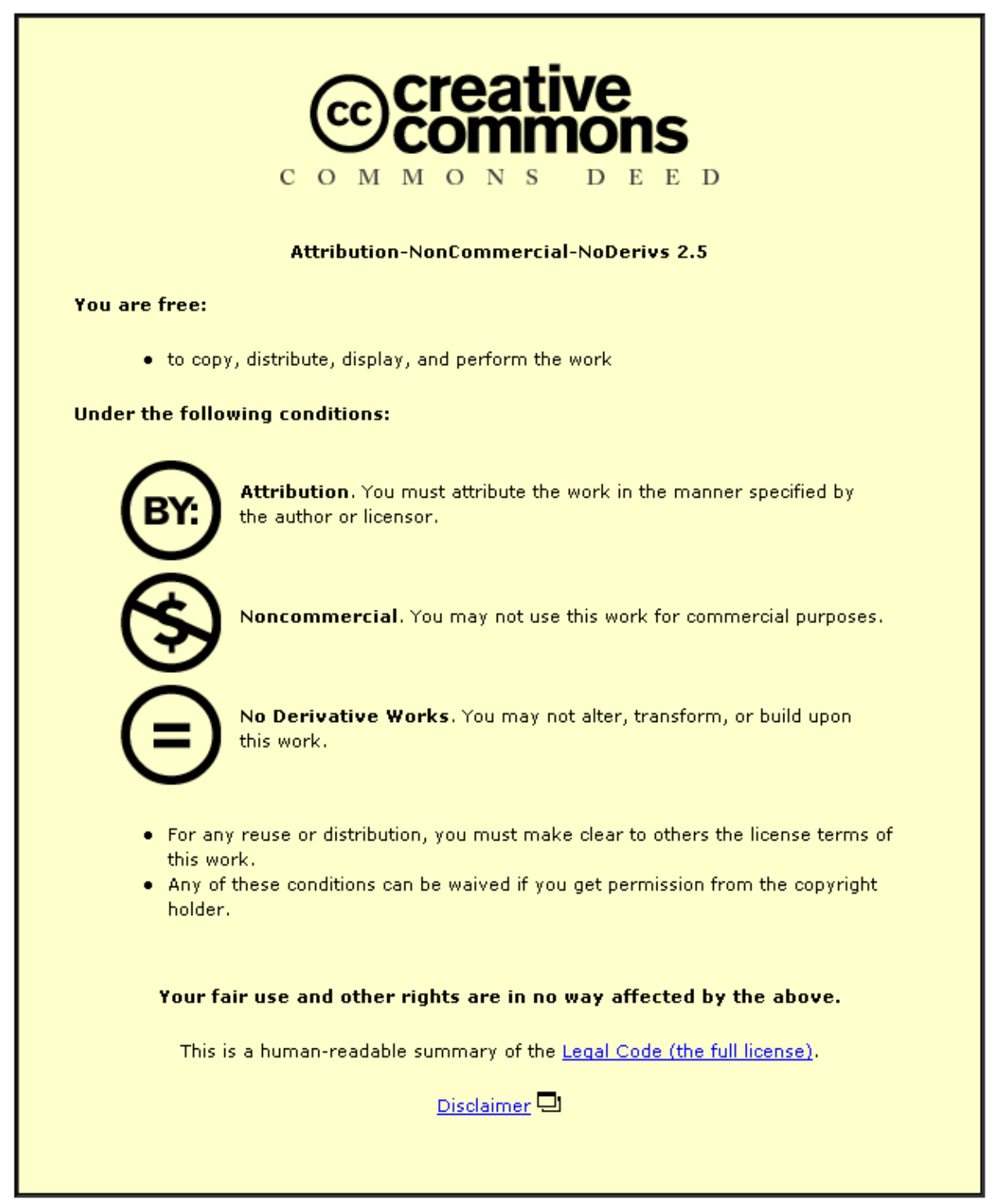

For the full text of this licence, please go to: http://creativecommons.org/licenses/by-nc-nd/2.5/ 
Sports Biomechanics 8, 39-51, 2009

\title{
Optimisation of the felge on parallel bars
}

\author{
${ }^{1}$ Michael J Hiley, ${ }^{2}$ Roger Wangler and ${ }^{1}$ Gheorghe Predescu
}

\footnotetext{
${ }^{1}$ School of Sport, Exercise \& Health Sciences, Loughborough University, Loughborough, LE11 3TU, UK

${ }^{2}$ Swiss Federal Institute of Technology ETH Zurich, Zurich, Switzerland
}

\begin{abstract}
The felge, or undersomersault, to handstand on parallel bars has become an important skill in Men's Artistic Gymnastics as it forms the basis of many complex variations. To receive no deductions from the judges, the felge must be performed without demonstrating the use of strength to achieve the final handstand position. Two male gymnasts each performed nine trials of the felge from handstand to handstand while data were recorded using an automatic motion capture system. The highest and lowest scoring trials of each gymnast, as determined by four international judges, were chosen for further analysis. The technique used by each gymnast was optimised using a computer simulation model so that the final handstand position could be achieved with straight arms. Two separate optimisations found different techniques identified in the coaching literature that are used by gymnasts. Optimum simulations resulted in improved performances through a combination of increased vertical velocity and height of the mass centre at release. Although the optimum technique found close to the gymnasts' own technique was more demanding in terms of the strength required, it offered the potential for more consistent performance and future developments in skill complexity.
\end{abstract}

\section{INTRODUCTION}

In the new Code of Points (Fédération Internationale de Gymnastique (FIG), 2006) the felge, or undersomersault, on parallel bars has become an important skill in Men's Artistic Gymnastics as it forms the basis for many variations of the skill. Although the basic skill is performed to support, it is the felge from handstand to handstand (Figure1) that provides the basis for the more complex variations. From the handstand position the gymnast lowers the body by closing the shoulder angle (shoulder extension) and allowing the shoulders to move forwards relative to the hands (Figure 2). The gymnast then rotates backwards about the point of contact with the bars and circles below the bar. Release occurs shortly after the mass centre has passed above the level of the bars (Figures $1 \& 2$ ). The gymnast re-grasps the bars before reaching the handstand position. In order to receive no deductions from the judges, the gymnast must perform the felge without demonstrating the use of strength to achieve the final handstand position. The gymnast should arrive in the final position without bending the arms or appearing to press to handstand (excessively flexing at the shoulder once the bar has been caught).

The technique depicted in the Code of Points (FIG, 2006), Figure 1, closely resembles a backward clear circle to handstand as performed on the high bar. During this technique the gymnast maintains the hip flexion angle throughout the majority of the circle, in particular whilst the gymnast is below the bars (Figure 1). It has been recommended that this technique is used during the initial stages of learning the felge (Davis, 2005), presumably because it is less demanding of the young gymnasts. However, the technique used by many senior gymnasts more closely resembles a "stoop stalder" (Davis, 2005). As the gymnast passes beneath the bars a deep pike position (large hip flexion angle) is adopted from which the gymnast rapidly extends passing through release and into the final handstand position (Figure 2). One of the problems encountered during the felge is that as the mass centre passes above the bars the 
gymnast's grip on the bars is reduced. With a reduced grip, the gymnast is less able to press against the bars and forcibly open the shoulder angle to generate the necessary vertical velocity of the mass centre. It is speculated that in the stoop stalder technique the gymnast uses rapid hip extension earlier in the circle, whilst the grip on the bar is strong, to generate the required vertical velocity. Such a technique, however, may require greater joint torques.

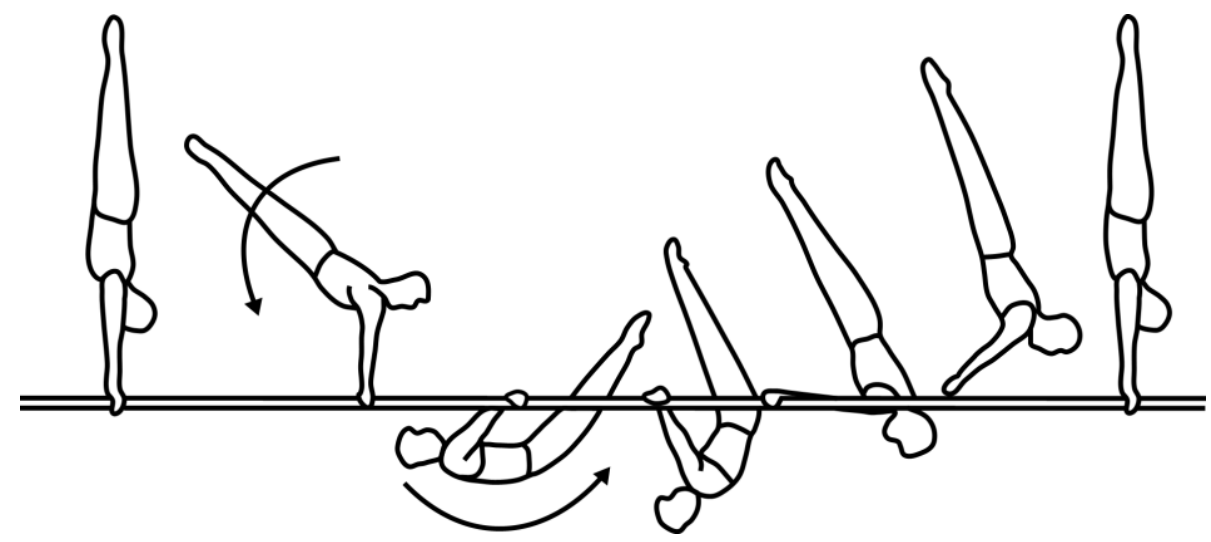

Figure 1. The felge from handstand to handstand on parallel bars (adapted from the FIG Code of Points, 2006).

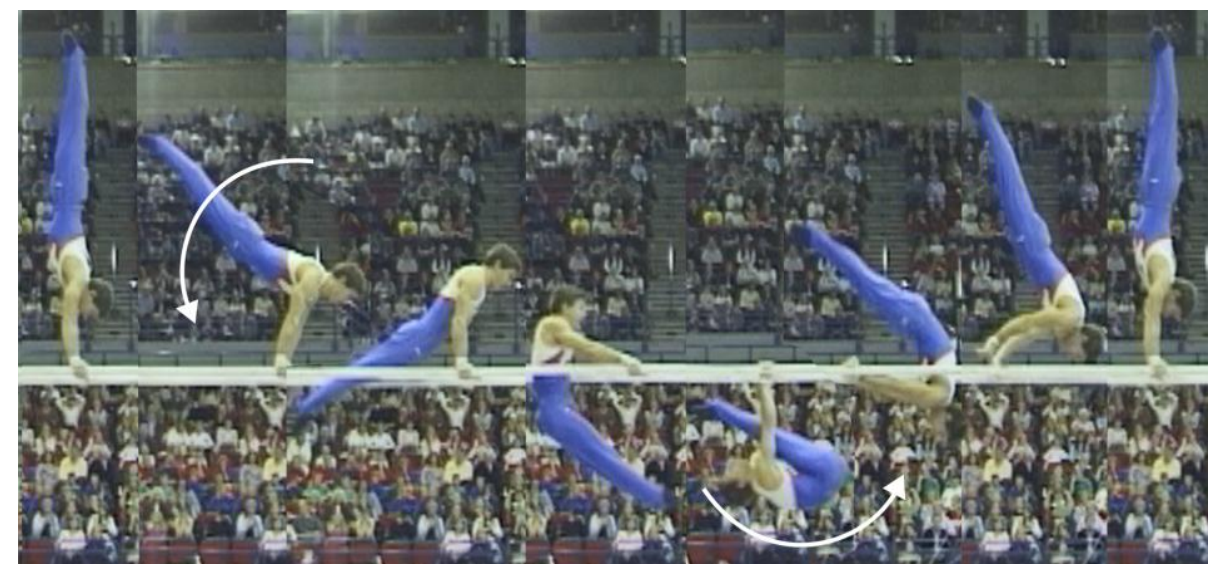

Figure 2. The flege from handstand to handstand performed with a "deep" hip flexion as the gymnast passes beneath the bars.

Previous research has investigated the felge to handstand mount on parallel bars (Takei and Dunn, 1996). The mount starts from a standing position from which the gymnast jumps into the swing. The performances of 26 national gymnasts were split into two groups by score and then analysed. It was found that the better performances were associated with a higher mass centre position and vertical velocity at release. It was also found that in the poorer performances the gymnasts over-rotated before releasing the bars which lead to a poor body position on re-grasp. Being over-rotated at release would result in the gymnast re-grasping the bars with a larger angle between the body and the vertical. To achieve the handstand from this position the gymnast would be required to press to handstand, incurring deductions from the judges. 
In order to investigate which is the best technique, computer simulation modelling could be used as it allows many variations in technique to be tried without risking injury to the gymnast. Limited work has been carried out on simulating swinging on parallel bars. A two-dimensional frontal plane model of the gymnast and bars has been used to look at the lateral movements of the bars during handstand (Linge et al., 2006). The model was evaluated using data collected from a pendulum oscillating in the frontal plane and was then used to simulate a gymnast re-grasping the bars in the handstand position from a flighted backward somersault. Although the model was able to simulate the lateral movements of the bars during these activities no data or results were presented for swinging in the sagittal plane. It might be expected that there will be lateral movements of the bars during the felge to handstand, although these are likely to be relatively small $(<0.02 \mathrm{~m})$ and symmetrical about the sagittal plane. Therefore a two-dimensional sagittal plane model of swinging on parallel bars would seem to be appropriate to investigate improving a gymnast's technique.

The aim of the present study was (a) to optimise the existing technique of gymnasts performing the felge from handstand to handstand so that the final position could be achieved with straight arms and without excessive pressing to handstand and (b) to investigate to what extent minimisation of joint torque can explain the technique used by gymnasts. An additional aim was to determine the relative advantages of the two techniques described above (i.e. the stoop stalder and the clear circle).

\section{METHODS}

Subsections in Methods describe the protocol used to optimise the technique in the felge from handstand to handstand on parallel bars. Initially data collection was carried out in which performances were recorded using a three-dimensional motion capture system and the data were processed for subsequent use with a computer simulation model. The model was used to obtain matching simulations of the actual performances in order to obtain model parameters. The matching simulations were then used as the starting point to optimise the gymnast's technique in order to achieve the final handstand position with straight arms.

\section{Data collection}

Two senior male gymnasts competing at national level (subject 1 : mass $=61.2 \mathrm{~kg}$, height $1.65 \mathrm{~m}$; subject 2: mass $=63.5 \mathrm{~kg}$, height $1.75 \mathrm{~m}$ ) each performed 9 trials of the felge from handstand to handstand. All trials were captured using 13 Vicon M2 cameras operating at $100 \mathrm{~Hz}$ (reconstruction error for the calibration of the motion capture system was less than $0.002 \mathrm{~m}$ ). Spherical reflective markers $25 \mathrm{~mm}$ in diameter were attached to the lateral side of the wrist, elbow, shoulder, hip, knee and ankle joint centres and toes on each side of the body. Offset measurements from each marker centre to the adjacent joint centre were recorded using an anthropometer for subsequent determination of the joint centres. Additional markers were attached to each side of the gymnast's head and on top of each parallel bar at its centre. In addition to the Vicon cameras all trials were recorded with a standard $50 \mathrm{~Hz}$ digital video camcorder (Panasonic NV-GS200EB). Prior to data collection a volume centred on the parallel bars spanning approximately $5 \mathrm{~m} \times 5$ $\mathrm{m} \times 5 \mathrm{~m}$ was wand calibrated using the Vicon motion analysis system. Threedimensional marker coordinates were reconstructed and joint centres were calculated using the measured offsets from which arm orientation and joint configuration angles 
were calculated (Yeadon, 1990a). A set of 95 anthropometric measurements were taken on each gymnast and inertia parameters were calculated using the model of Yeadon (1990b).

Four judges with international accreditation (FIG) scored each felge from the video recordings. The highest and lowest scoring trials of each gymnast were chosen for further analysis. None of the chosen trials achieved the final handstand position with straight arms, using instead a combination of bent arms and pressing to handstand.

\section{Simulation model}

A four-segment planar model of a gymnast comprising arm, torso, thigh and lower leg segments was used to simulate the swinging movement on the parallel bars (Hiley and Yeadon, 2003a). Since flexing of the elbow or knee would lead to deductions from the judges, a single segment was sufficient to represent straight arms and the legs were constrained to be straight. The bar and the gymnast's shoulder structure were modelled as damped linear springs (Figure 3). The shoulder spring represented the stretch at the shoulder and any extension of the spine that may occur. The movement of the shoulder (gleno-humeral) joint centre due to scapular rotation was represented by allowing the torso length to vary as a function of shoulder (arm elevation) angle $\alpha$ (Figure 3 ).

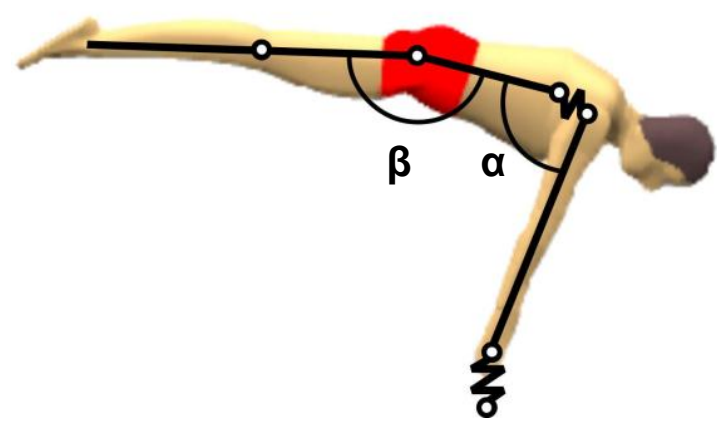

Figure 3. Four segment simulation model of the gymnast and parallel bars.

Input to the simulation model comprised the segmental inertia parameters, the stiffness and damping coefficients of the bar and shoulder springs, the initial displacement and velocity of the bar, the initial orientation and angular velocity of the arm, and the joint angle time histories at the hip and shoulder in the form of Fourier series. Output from the model comprised the time histories of the bar displacements, the linear and angular momentum of the model and the rotation angle $\phi$ (the angle from the upward vertical of the line joining the neutral bar position to the mass centre).

The equations of motion were derived using Newton's Second Law and by taking moments about the neutral bar position and the segment mass centres (Hiley and Yeadon, 2003a). The angular momentum of the body about its mass centre at release was normalised by dividing by $2 \pi$ times the moment of inertia of the body about its mass centre when straight, giving the number of straight somersaults per second (ss/s). 


\section{Matching Simulations}

In order to determine model parameters and provide a starting point for the optimisation of technique a close match between simulated and recorded performance was required for the two selected trials of each gymnast. The simulation model was implemented with the Simulated Annealing optimisation algorithm (Goffe et al., 1994) to minimise the difference between the recorded and simulated performance. The cost function used included terms for differences between recorded and simulated rotation angle, bar displacements and joint angle time histories at the hip and shoulder, normalised angular momentum at release and linear velocity at release (Table 1). The method for obtaining the data from the actual performances is described in Hiley and Yeadon (2003b).

Since the model was designed to simulate the swinging portion of the skill each simulation was started once the angular velocity of the arm segment was in the positive direction (anticlockwise, Figure 2). The end of the simulation was defined as the point at which the gymnast lost contact with the bars. The previously determined subject-specific inertia parameters were used in the simulation model. The initial conditions, including the initial angle, angular velocity and bar displacements, for each simulation were taken from the corresponding trial. During the optimisation the following parameters were allowed to vary in order to improve the match between the recorded and simulated performance. The vertical bar stiffness was allowed to vary between $40,000 \mathrm{~N} / \mathrm{m}$ and $90,000 \mathrm{~N} / \mathrm{m}$ to conform with the specifications of the International Gymnastics Federation (FIG, 2006) and the vertical damping coefficient of the bar was allowed to vary between $0 \mathrm{~N} \cdot \mathrm{s} / \mathrm{m}$ and $1000 \mathrm{~N} \cdot \mathrm{s} / \mathrm{m}$. The horizontal bar stiffness was allowed to vary between $75,000 \mathrm{~N} / \mathrm{m}$ and $200,000 \mathrm{~N} / \mathrm{m}$ and the horizontal damping coefficient of the bar was allowed to vary between $0 \mathrm{~N} \cdot \mathrm{s} / \mathrm{m}$ and $10,000 \mathrm{~N} \cdot \mathrm{s} / \mathrm{m}$. The horizontal stiffness of the bar was much larger than the vertical stiffness since the bars were constrained to minimal movement in the horizontal direction due to the construction of the apparatus. The stiffness and damping coefficients of the shoulder spring were allowed to vary over wide ranges, between $0 \mathrm{~N} / \mathrm{m}$ and $30,000 \mathrm{~N} / \mathrm{m}$ and $0 \mathrm{~N} \cdot \mathrm{s} / \mathrm{m}$ and $5,000 \mathrm{~N} \cdot \mathrm{s} / \mathrm{m}$, respectively, since there was less information available regarding these parameters. The masses of the arms and legs were allowed to vary independently by $\pm 5 \%$ and the torso mass was adjusted to maintain whole body mass, since density values were taken from the literature for the segmental mass calculations. The torso length parameter was allowed to vary between $0 \mathrm{~m}$ and $0.15 \mathrm{~m}$ (Begon et al., 2008). In addition small variations in the initial conditions, rotation angle and angular velocity were permitted to compensate for any errors propagated in their calculation $( \pm 0.1 \mathrm{rad}$ and $\pm 1.0 \mathrm{rad} / \mathrm{s}$, respectively). Finally, the coefficients which defined the Fourier series were allowed to vary to obtain a close match with the recorded joint angle time histories.

\section{Optimisations}

The cost function optimised was based on minimising the joint torques at the hip and shoulder joints whilst seeking an acceptable felge through the use of appropriate penalties. From the matching simulations peak joint torques for flexion and extension at each joint were determined. The joint torque limits during optimisation were based on these values (Table 2). In addition a reduced limit based on the results of the matching simulations was placed on the joint torques (Table 2) once the model had rotated through $250^{\circ}$ to reflect the reduced grip on the bars. The simulations started from the same point as in the matching process and finished once the torso segment had rotated $40^{\circ}$ past the 
vertical, since release should occur before this point in a good performance (Takei and Dunn, 1996). The cost function was calculated from when the torso segment reached the vertical through to $40^{\circ}$ past the vertical. The value returned to the optimisation was the lowest value of the cost function obtained during this period. The matching simulations were used to give the initial estimates of the joint angle time histories for the four optimisations. The upper and lower bounds for the Fourier series coefficients were allowed to vary by $\pm 25 \%$ from the initial values. The optimum technique was required to produce sufficient vertical velocity at release to achieve a mass centre height in flight of at least $90 \%$ of the final handstand position measured above the bars. The $90 \%$ of handstand position was chosen as gymnasts re-grasp the bars before handstand is reached. It also represented a marked improvement over the actual performances (on average an improvement of $21 \%$ and $28 \%$ for subjects 1 and 2 , respectively - Table 1). The vertical velocity required to reach $90 \%$ of handstand was calculated from the height of the mass centre at release and in the final handstand position using the equations of motion under constant acceleration. The simulation also incurred penalties if the horizontal velocity and normalised angular momentum at release exceeded the range obtained from the video analysis of the 18 trials and the values reported for high scoring performances by Takei and Dunn (1996) $(0.3 \mathrm{~m} / \mathrm{s}>$ horizontal velocity $>-0.2 \mathrm{~m} / \mathrm{s}$ and $0.025 \mathrm{ss} / \mathrm{s}>$ normalised angular momentum $>-0.025 \mathrm{ss} / \mathrm{s})$. A further penalty was imposed for excessive hip flexion angles at release from the bars as this was likely to result in poor body configurations on re-grasping the bars (Takei and Dunn, 1996).

The optimisations were run twice: the first set of four optimisations with no limits placed on the joint angle time histories (other than those described above) and the second set where they were constrained to produce a stoop stalder technique - this was achieved by creating a penalty for flexing too early in the felge and not producing the characteristic deep pike position.

\section{RESULTS}

The information from the motion capture analysis gave the following results. The release parameters for the four performances are given in Table 1 along with the height of the mass centre at re-grasp and in the final handstand position (relative to bar height).

Table 1. Release parameters for the four felge to handstand performances

\begin{tabular}{lcccc}
\hline \multicolumn{1}{c}{ Variable } & \multicolumn{2}{c}{ Subject 1 } & \multicolumn{2}{c}{ Subject 2 } \\
& Best trial & Worst trial & Best trial & Worst trial \\
\hline $\mathrm{CM}_{\mathrm{h} \text { release }(\mathrm{m})}$ & 0.177 & 0.187 & 0.176 & 0.145 \\
$\mathrm{CM}_{\mathrm{h} \text { re-grasp }(\mathrm{m})}$ & 0.642 & 0.595 & 0.614 & 0.656 \\
$\mathrm{CM}_{\mathrm{h} \text { handstand }}(\mathrm{m})$ & 0.902 & 0.902 & 1.018 & 1.018 \\
Vert velocity release $(\mathrm{m} / \mathrm{s})$ & 3.115 & 2.902 & 3.171 & 3.169 \\
Horiz velocity release $(\mathrm{m} / \mathrm{s})$ & 0.097 & 0.164 & 0.035 & 0.319 \\
Angular momentum $(\mathrm{ss} / \mathrm{s})$ & 0.000 & 0.007 & -0.004 & -0.002 \\
\hline
\end{tabular}

Note: $\mathrm{CM}_{\mathrm{h}}$ is mass centre height

Note: Angular momentum is measured in straight somersaults (ss) per second 
Over the approximate $270^{\circ}$ rotation of the four matching simulations the model was able to reproduce the whole body rotation angle to within $2^{\circ}$ root mean squared (rms) difference and the displacements of the bar to within $0.005 \mathrm{~m}$ rms difference (Figure 4). The matches between the measured joint angle time histories from the video analysis and those determined using Fourier series were close with an average rms difference of $4^{\circ}$ (Figure 4c). The simulation model matched the mass centre velocity at release to less than $1 \%$. The mean vertical stiffness and damping coefficients of the bar obtained in the four matching trials were $63,573 \mathrm{~N} / \mathrm{m}$ (standard deviation $1,041 \mathrm{~N} / \mathrm{m}$ ) and $686 \mathrm{~N} \cdot \mathrm{s} / \mathrm{m}( \pm$ $58 \mathrm{~N} \cdot \mathrm{s} / \mathrm{m}$ ), respectively. The horizontal stiffness and damping coefficients of the bar were considerably larger, due to the construction of the apparatus $(152,189 \mathrm{~N} / \mathrm{m} \pm$ $10,052 \mathrm{~N} / \mathrm{m}$ and $7,880 \mathrm{~N} \cdot \mathrm{s} / \mathrm{m} \pm 1824 \mathrm{~N} \cdot \mathrm{s} / \mathrm{m})$. The average shoulder stiffness and damping coefficients were $14,308 \mathrm{~N} / \mathrm{m}( \pm 2,018 \mathrm{~N} / \mathrm{m})$ and $1,656 \mathrm{~N} \cdot \mathrm{s} / \mathrm{m}( \pm 42 \mathrm{~N} \cdot \mathrm{s} / \mathrm{m})$, respectively. The peak joint torques at the hip and shoulder during the matching simulations are given in Table 2.

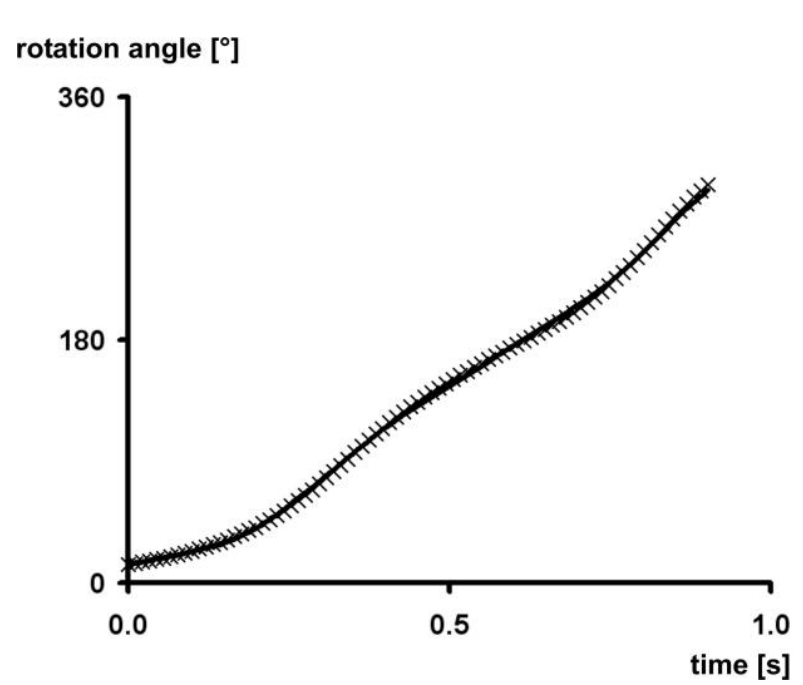

(a)

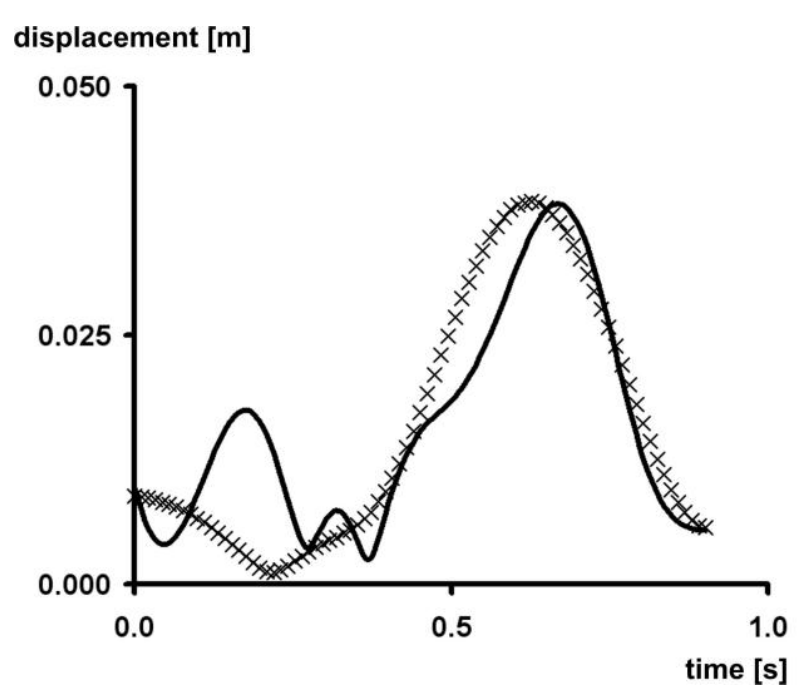

(b)

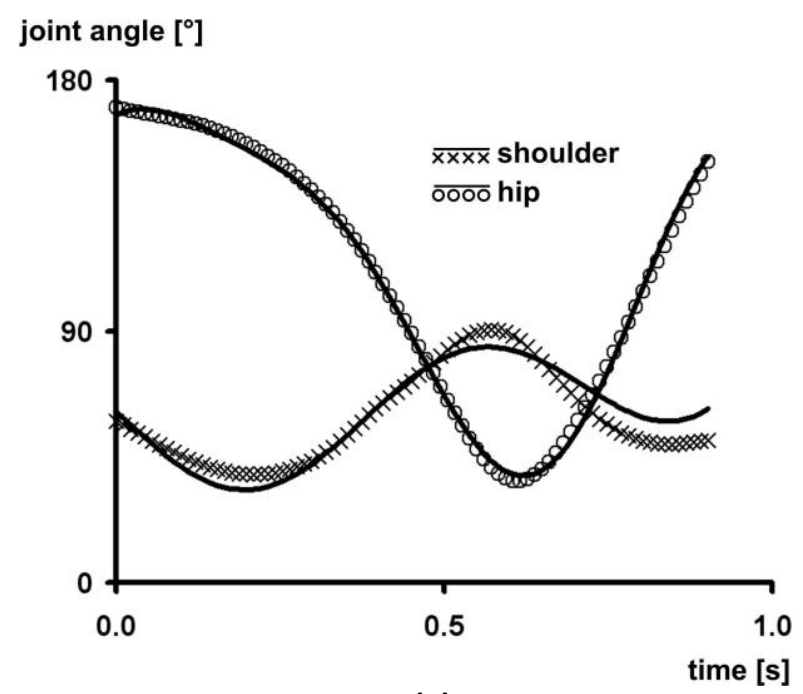

(c)

Figure 4. Typical matches between simulation (solid line) and actual performance (crosses) of the bar contact phase of the felge to handstand for (a) whole body rotation angle, (b) net bar displacement and (c) joint angle time histories of the shoulder and hip. 
Table 2. Peak joint torques for the four felge to handstand performances and the joint torque limits used in the optimisations

\begin{tabular}{|c|c|c|c|c|}
\hline \multirow[b]{2}{*}{ Variable } & \multicolumn{2}{|c|}{ Subject 1} & \multicolumn{2}{|c|}{ Subject 2} \\
\hline & Best trial & Worst trial & Best trial & Worst trial \\
\hline Peak shoulder torque (max / $\min , \mathrm{Nm}$ ) & $374 /-427$ & $402 /-377$ & $354 /-169$ & $429 /-212$ \\
\hline Peak hip torque ( $\max / \min , \mathrm{Nm})$ & $93 /-209$ & $191 /-398$ & $213 /-469$ & $184 /-460$ \\
\hline Shoulder torque limit (max / $\min , \mathrm{Nm})$ & \multicolumn{2}{|c|}{$405 /-430$} & \multicolumn{2}{|c|}{$430 /-215$} \\
\hline Hip torque limit (max / min, Nm) & \multicolumn{2}{|c|}{$200 /-400$} & \multicolumn{2}{|c|}{$215 /-470$} \\
\hline Shoulder torque limit release $(\mathrm{Nm})$ & \multicolumn{2}{|c|}{ \pm 175} & \multicolumn{2}{|c|}{ \pm 175} \\
\hline Hip torque limit release (Nm) & \multicolumn{2}{|c|}{ \pm 200} & \multicolumn{2}{|c|}{ \pm 200} \\
\hline
\end{tabular}

In the first set of optimisations where the joint angle time histories were not constrained the model was able to achieve the appropriate vertical velocity at release whilst satisfying the criteria for a successful performance. At release the model had a higher mass centre position and vertical velocity than in the actual performances. The release parameters and peak joint torques for the optimised simulations are given in Table 3. The peak joint torques from the optimisations were lower than in the actual performances and did not exceed any of the limits (Figure 5). Negative torques refer to hip extension and shoulder flexion (opening the joint angles). The technique in the first set of optimised simulations differed from the technique of the gymnasts (Figure 5, 6 and 7) and more closely resembled the clear circle technique.

Table 3. Release parameters and peak joint torques for the four optimised felge to handstand simulations

\begin{tabular}{lcccc}
\hline \multicolumn{1}{c}{ Variable } & \multicolumn{2}{c}{ Subject 1 } & \multicolumn{2}{c}{ Subject 2} \\
& Best trial & Worst trial & Best trial & Worst trial \\
\hline $\mathrm{CM}_{\mathrm{h} \text { release }(\mathrm{m})}$ & 0.196 & 0.201 & 0.249 & 0.274 \\
Vert velocity release $(\mathrm{m} / \mathrm{s})$ & 3.574 & 3.540 & 3.623 & 3.558 \\
Horiz velocity release $(\mathrm{m} / \mathrm{s})$ & -0.137 & 0.105 & -0.104 & -0.084 \\
Angular momentum $(\mathrm{ss} / \mathrm{s})$ & -0.025 & -0.023 & -0.017 & -0.019 \\
Max shoulder torque $(\mathrm{Nm})$ & 136 & 139 & 383 & 281 \\
Min shoulder torque $(\mathrm{Nm})$ & -124 & -179 & -138 & -126 \\
Max hip torque $(\mathrm{Nm})$ & 143 & 139 & 165 & 195 \\
Min hip torque $(\mathrm{Nm})$ & -206 & -195 & -200 & -226 \\
\hline
\end{tabular}

Note: Angular momentum is measured in straight somersaults (ss) per second 

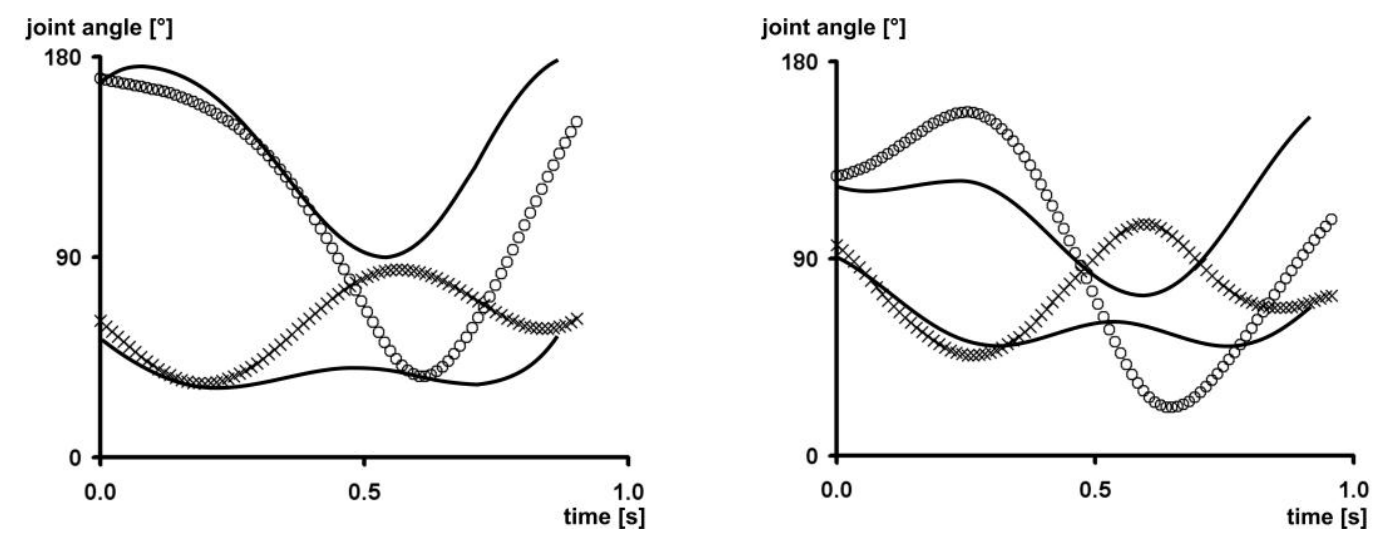

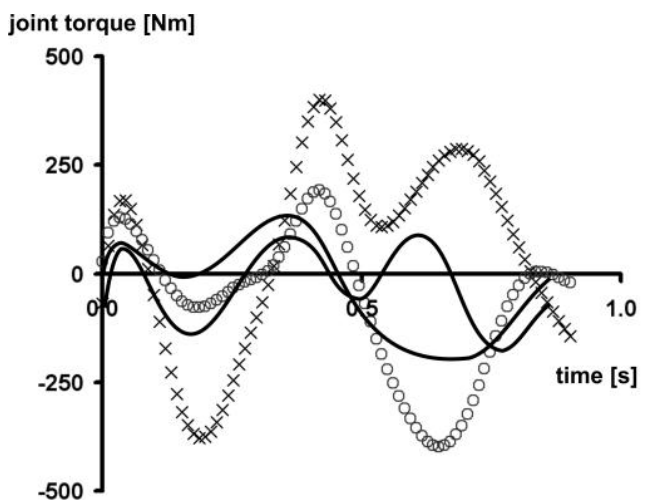

(a)

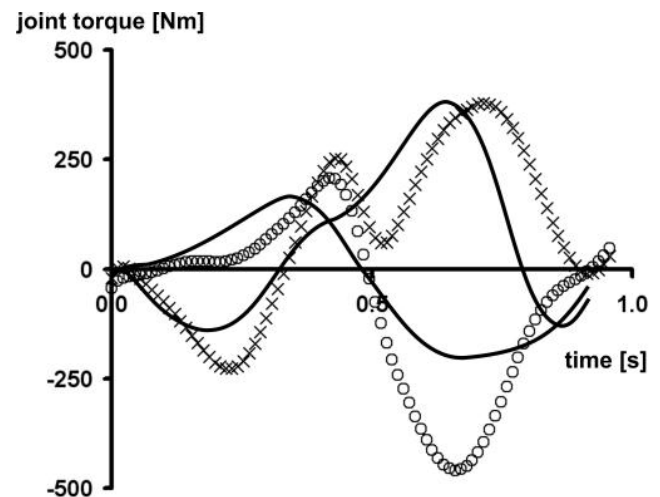

(b)

Figure 5. Joint angle and joint torque histories of the hip (circles) and shoulder (crosses) for the matched and optimised (solid line) simulations of (a) subject 1 worst trial and (b) subject 2 best trial.

(a)

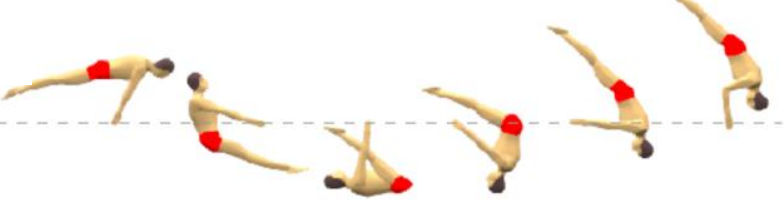

(b)

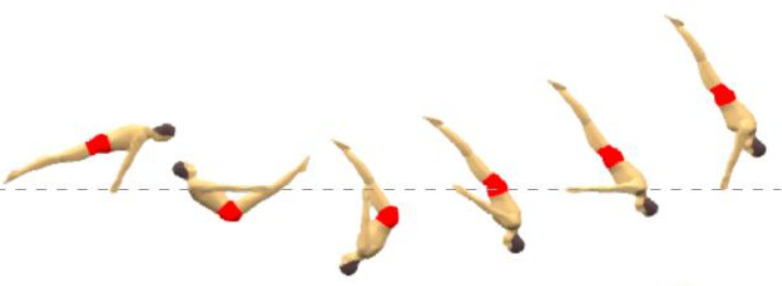

(c)

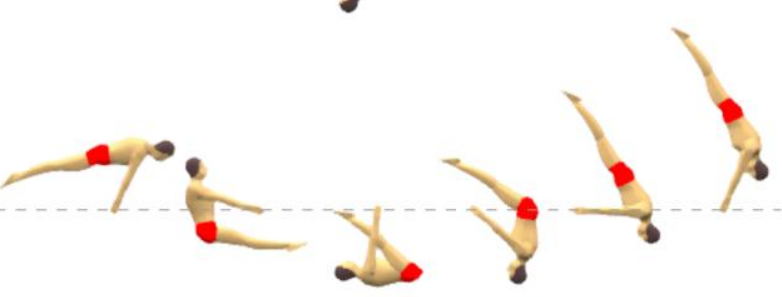

Figure 6. Graphical sequences of the (a) matched (re-grasp position taken from video analysis), (b) optimised and (c) constrained optimisation of subject 1 worst trial. 
(a)

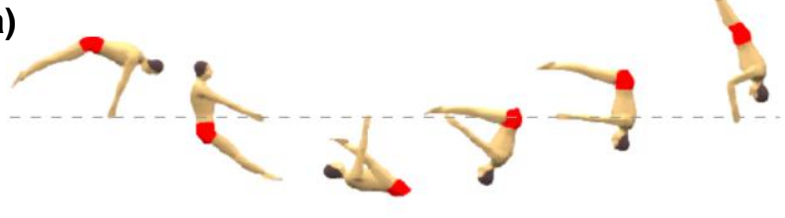

(b)

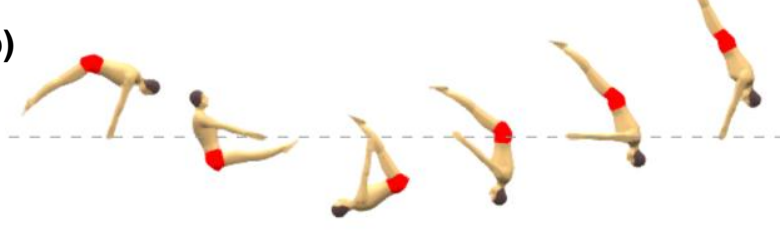

(c)

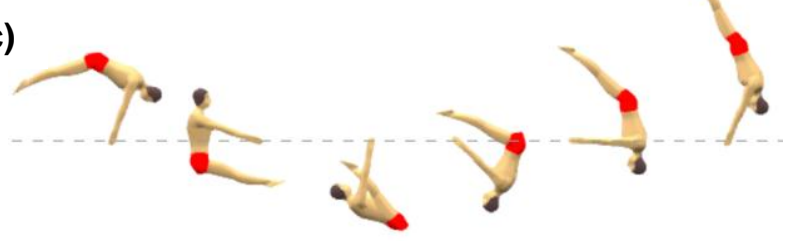

Figure 7. Graphical sequences of the (a) matched (re-grasp position taken from video analysis), (b) optimised and (c) constrained optimisation of subject 2 best trial.

In the optimisations where the joint angle time histories were constrained to produce a technique similar to the gymnasts' own technique the model was still able to achieve the appropriate vertical velocity at release whilst satisfying the criteria for a successful performance. However, although the peak joint torques did not exceed the limits set from the actual performances (highest torques obtained from each of the gymnasts' two trials) they were higher than those obtained from the first set of optimisations (Tables 3 and 4). The common feature of the optimal stoop stalder simulations was a more rapid hip extension over a slightly larger hip angle range, when compared with the actual performances (Figure 8). Graphical sequences of the constrained optimal simulations for each gymnast are given in Figures 6 and 7.

Table 4. Release parameters and peak joint torques for the four constrained optimial felge to handstand simulations

\begin{tabular}{lcccc}
\hline & \multicolumn{2}{c}{ Subject 1} & \multicolumn{2}{c}{ Subject 2} \\
Variable & Best trial & Worst trial & Best trial & Worst trial \\
\hline $\mathrm{CM}_{\mathrm{h} \text { release }(\mathrm{m})}$ & 0.179 & 0.242 & 0.271 & 0.329 \\
Vert velocity release $(\mathrm{m} / \mathrm{s})$ & 3.550 & 3.536 & 3.558 & 3.396 \\
Horiz velocity release $(\mathrm{m} / \mathrm{s})$ & -0.189 & -0.130 & -0.175 & -0.199 \\
Angular momentum $(\mathrm{ss} / \mathrm{s})$ & -0.022 & -0.020 & -0.011 & -0.021 \\
Max shoulder torque $(\mathrm{Nm})$ & 309 & 326 & 414 & 423 \\
Min shoulder torque $(\mathrm{Nm})$ & -308 & -387 & -201 & -179 \\
Max hip torque $(\mathrm{Nm})$ & 106 & 126 & 109 & 198 \\
Min hip torque $(\mathrm{Nm})$ & -396 & -369 & -419 & -433 \\
\hline
\end{tabular}

Note: Angular momentum is measured in straight somersaults (ss) per second 

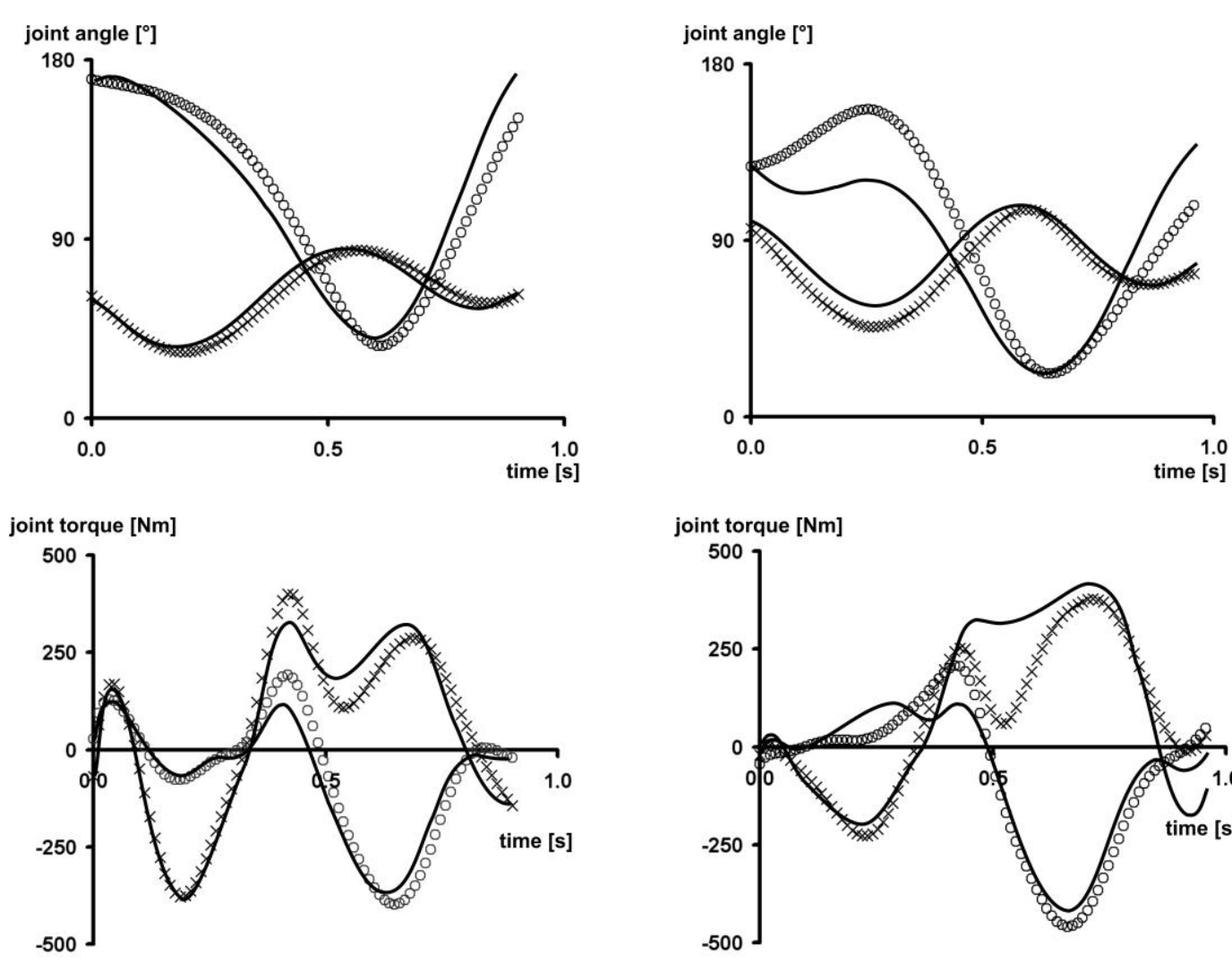

(a)

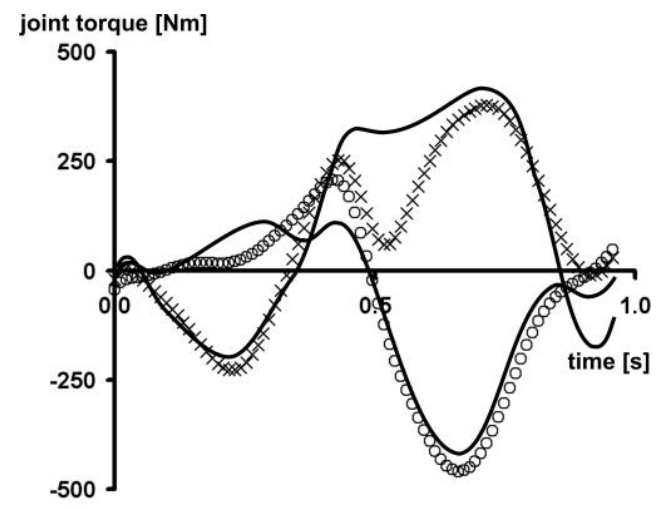

(b)

Figure 8. Joint angle and joint torque histories of the hip (circles) and shoulder (crosses) for the matched and constrained optimised (solid line) simulations of (a) subject 1 worst trial and (b) subject 2 best trial.

\section{DISCUSSION AND IMPLICATIONS}

In all four cases the first set of optimisations were able to improve the gymnasts' performances with the mass centre able to reach over $90 \%$ of handstand height in the flight phase. The average heights achieved by the two gymnasts in their actual performances were $72 \%$ and $66 \%$ of handstand, respectively. The improvement was achieved through a combination of increased vertical velocity and an increase in the height of the mass centre at release (Tables 1, 3 and 4). However, the optimised techniques were not representative of the gymnast's actual performances. The technique changed from the stoop stalder to one that more closely represented the clear circle technique. The main characteristics of this technique are achieving the peak hip flexion angle earlier in the circle and having a less flexed hip angle (Figures 5, 6b and 7b). It may be argued that given the cost function the clear circle technique was the global optimum, since it was found when no constraints were placed on the joint angle time histories. However, the aim was to optimise the gymnasts' technique rather than find an alternative optimum solution.

Therefore additional joint angle constraints were imposed to obtain an optimal solution which was similar to the gymnasts' technique. When the optimisation used a constraint to encourage a stoop stalder technique the second set of optimal solutions were still able to achieve the required increase in vertical velocity at release. The increased vertical velocity at release was produced predominantly by a more rapid extension of the hip angle (Figure 8). The hip angle was also extended over a larger range (Figures 6c, 7c and 8 ) which also lead to an increase in mass centre height at 
release. The higher mass centre location and more extended body configuration at release have been shown to be desirable from a judging perspective (Takei and Dunn, 1996).

Both sets of optimisations were able to achieve the improved performance whilst staying within the joint torque limits defined by the gymnasts' actual performances. It might therefore be expected that the level of improvements seen in the optimisations could be achieved by the gymnasts. When choosing which technique to use, it can be seen from Tables 3 and 4 that in terms of peak joint torque the stoop stalder is more demanding of the gymnast. This is why in the early stages of learning, the clear circle technique is adopted, as recommended by Davis (2005). The clear circle technique may be characterised as a global optimum arising from minimisation of joint torque. Since this is the case, why then is the stoop stalder technique adopted by the majority of senior gymnasts? It has been demonstrated here that it requires more strength, so what benefits does it offer the gymnast compared with the clear circle technique?

The path of the mass centre during the optimal felges is shown in Figure 9. In the optimisations encouraged to produce the stoop stalder the path of the mass centre is flatter and more vertical as the gymnast approaches release. This has two advantages: firstly the direction of the mass centre velocity changes less near to release, when compared to the clear circle technique, and this should lead to a more consistent performance. If the release is mistimed the mass centre will still be moving in the correct direction. This has been shown to be the case for other release skills in gymnastics (Hiley and Yeadon, 2003b). Secondly, the felge to handstand forms the basis of more complex skills, typically the felge to handstand with either a half or full twist. In these skills there is not a flight phase as such, rather the gymnast makes hand changes whilst the force on the bars is low. Having a vertical mass centre velocity while the body is twisting reduces the task complexity of the hand changes (i.e. less correction for non vertical alignment). Had the requirement of a near vertical path of the mass centre prior to release been included in the cost function, it might be expected that the stoop stalder would have arisen as a global optimum.

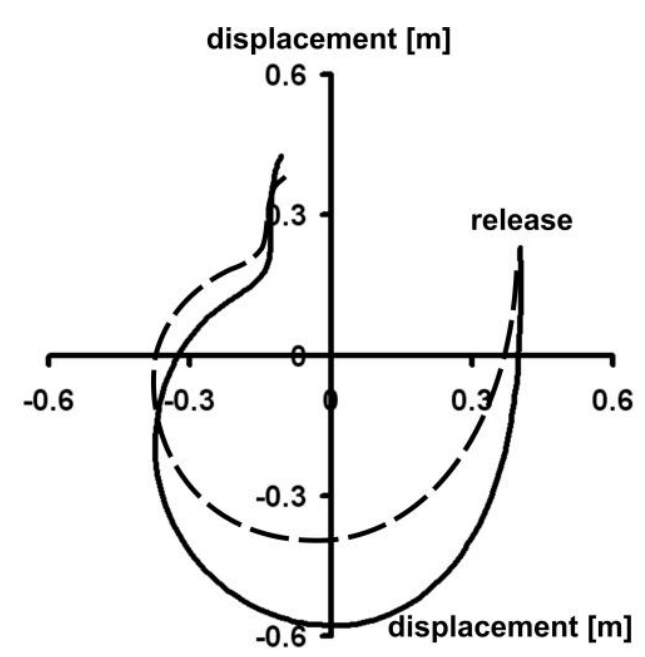

(a)

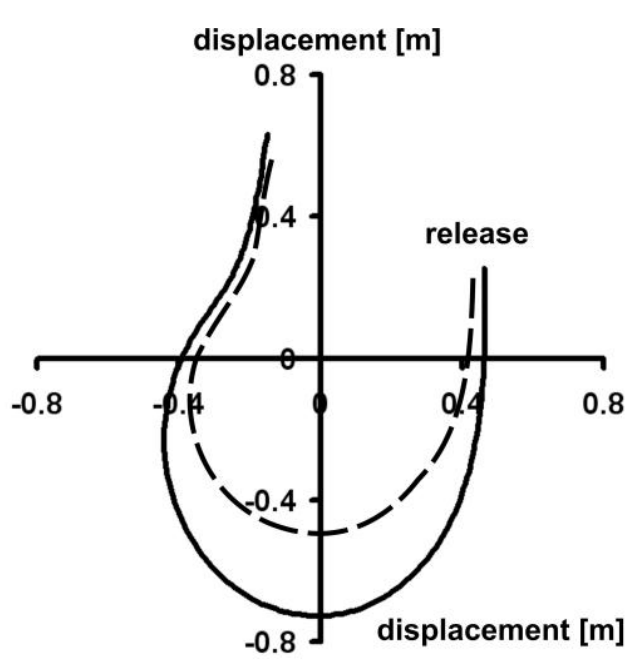

(b)

Figure 9. Path of the mass centre for the optimised (dashed line) and constrained optimisation (solid line) for (a) subject 1 worst trial and (b) subject 2 best trial. 
One of the limitations of the present study is the use of the fixed joint torque limits. Although it would be desirable to have limits based on a joint torque - angle - angular velocity relationship it can be seen that the results do not depend on excessively large joint torques compared with the actual performances (Figures 5 and 8). Inspection of the joint angular velocity time histories revealed that peak angular velocity occurred at periods of low joint torque values. Inclusion of a torque - angle - angular velocity relationship may make small changes to the optimal technique, but it is not expected to affect the conclusions regarding the two techniques. A further limitation of the model was the single segment used to represent the spine. During large hip flexion angles there will be associated spinal flexion. In the present model both spinal and hip flexion were included at the hip. In future models spinal flexion could be represented as a function of hip flexion.

\section{CONCLUSIONS}

It was found that both good and poor performances of the felge from handstand to handstand could be improved. The technique used by the gymnasts could be improved by extending the hip angle more rapidly and over a larger range. The minimisation of joint torque resulted in a global optimum similar to the clear circle technique. Since the strength requirements of the clear circle are lower than those of the stoop stalder the clear circle is more appropriate for the early stages of development as suggested by Davis (2005). Although the optimum technique that closely resembled the technique used by the gymnasts was found to be more demanding in terms of the strength required, it does offer the potential for more consistent performance and future developments in skill complexity.

\section{References}

Begon, M., Wieber, P-B. and Yeadon, M.R. (2008). Kinematics estimation of straddled movements on high bar from a limited number of skin markers using a chain model. Journal of Biomechanics, 41, 581-586.

Davis, J. (2005). Undersomersaults on parallel bars. Gym Craft, 14, 6-7.

Fédération Internationale de Gymnastique, (2006). Code of Points. Moutier, Switzerland: F.I.G.

Goffe, W.L., Ferrier, G.D. and Rogers, J. (1994). Global optimization of statistical functions with simulated annealing. Journal of Econometrics, 60, 65-99.

Hiley, M.J. and Yeadon, M.R. (2003a). Optimum technique for generating angular momentum in accelerated backward giant circles prior to a dismount. Journal of Applied Biomechanics, 19, 119-130.

Hiley, M.J. and Yeadon, M.R. (2003b). The margin for error when releasing the high bar for dismounts. Journal of Biomechanics, 36, 313-319.

Linge, S., Hallingstad, O. and Solberg, F. (2006). Modelling the parallel bars in Men's Artistic Gymnastics. Human Movement Science, 25, 221-237.

Takei, Y. and Dunn, J.H. (1996). A comparison of techniques used by elite gymnasts in performing the basket-to-handstand mount. Journal of Sports Sciences, 14, 269-279. 
Yeadon, M.R. (1990a). The simulation of aerial movement - I. The determination of orientation angles from film data. Journal of Biomechanics, 23, 59-66.

Yeadon, M.R. (1990b). The simulation of aerial movement - II. A mathematical inertia model of the human body. Journal of Biomechanics, 23, 67-74. 